\section{Pectus updates and special considerations in Marfan syndrome}

\section{Stephanie Fraser, ${ }^{1}$ Anne Child, 2 Ian Hunt ${ }^{1}$}

1St. George's Hospital London;

2St. George's University of London, UK

\begin{abstract}
Congenital chest wall or pectus deformities including pectus excavatum (funnel chest) and pectus carinatum (pigeon chest) affect a significant proportion of the general population and up to $70 \%$ of patients with Marfan syndrome. Patients often experience significant morbidity and psychological distress, which can worsen with age. Here we discuss new techniques for both operative and non-operative treatment of pectus deformity, the importance of a welltimed intervention and special considerations in patients with Marfan syndrome.
\end{abstract}

\section{Introduction}

Pectus deformities including pectus excavatum (funnel chest) and pectus carinatum (pigeon chest) constitute the most common congenital anterior chest wall deformities. They affect a significant proportion of the general population $(0.8 \%)$ and an even greater proportion of patients with Marfan syndrome (up to $70 \%$ ). ${ }^{1}$ They are associated with a significant degree of morbidity and psychological distress; however, recent advances have highlighted several safe and well tolerated treatment options. Here we discuss both operative and non-operative techniques for the treatment of pectus deformity, the importance of a well-timed intervention and special considerations in patients with Marfan syndrome.

\section{Background}

Whilst in Marfan syndrome, pectus can be considered as integral part of the dominantly inherited deficiency of Fibrillin-1, in the general population, it is largely considered a primary hereditary connective tissue disorder. ${ }^{2}$ The exact pattern of inheritance has not been fully elucidated; however, familial studies suggest that this is likely to be complex and multifactorial. ${ }^{3}$ This would certainly be consistent with the plethora of clinical manifestations and phenotypes.
The common clinical features include abnormal development of the rib cage. Abnormal growth and elongation of the hyaline cartilaginous connection between the ribs and breastbone, known as the costal cartilage, results in either compression or protrusion of the breastbone (sternum). Unbalanced growth leads to asymmetry and may also involve misalignment and rotation of the sternum or sternal tilt. The deformity is sometimes visible in early childhood, but becomes more obvious during accelerated periods of growth, typically during puberty and early teenage years.

Many descriptions of the anatomical variations of pectus deformity exist, but most describe abnormal cartilages. A number of authors have suggested abnormal collagen content as the root cause, and indeed, histological studies have demonstrated an immature collagen matrix in costal cartilage of affected children. 4

This abnormal growth results in costal cartilages, which can be elongated, rotated, deformed and fused. Sternal angulation and rib or costal flaring or flattening are additional common sequelae.

Deformities are classified as either excavatum (depression) or carinatum (protrusion) though mixed deformities are also seen. Practically, the visual description of these deformities as either mild, moderate or severe (Figure 1), is most commonly in use, however, detailed classification systems of chest wall abnormalities exist. 5 Whilst the severity of the deformity does not necessarily imply that the patient will be more symptomatic, classification can be useful in communication between healthcare professionals and in decision-making regarding operative approach.

\section{Symptoms}

The anatomical abnormalities associated with pectus deformity are well described, but the physiological impact of these is less clear. Despite the lack of a clear pathological link, cadaveric studies suggest that patients with pectus excavatum have a shorter life expectancy. ${ }^{6}$

Symptoms commonly described by patients particularly those with pectus excavatum include breathlessness, chest pain on exertion, palpitations, an inability to perform physical activities at the same level of intensity as their peers and occasionally, syncope or pre-syncope during exercise.

Imaging studies and cardiac investigations in severe forms of pectus excavatum have demonstrated compression of intrathoracic structures leading to: reduced tho-
Correspondence: Ian Hunt, St. George's Hospital, Blackshaw Road, London, UK. Tel.: +44.208.725.3287 - Fax: +44.208.725.0068. E-mail: ian.hunt@stgeorges.nhs.uk

Key words: Pectus excavatum; pectus carinatum; Marfan; bracing; surgery.

Acknowledgements: we would like to acknowledge Pectus Services Ltd. for the use of their data

Contributions: the authors contributed equally.

Conflict of interest: the authors declare no potential conflict of interest

Received for publication: 14 May 2017

Revision received: 2 October 2017.

Accepted for publication: 20 October 2017.

This work is licensed under a Creative Commons Attribution NonCommercial 4.0 License (CC BY-NC 4.0).

CCopyright S. Fraser et al., 2017

Licensee PAGEPress, Italy

Pediatric Reports 2017; 9:7227

doi:10.4081/pr.2017.7227

racic volume, cardiac impingement or displacement, conduction abnormalities and valvular disease.7,8 Research has also demonstrated an association between pectus excavatum and impaired cardiovascular performance. ${ }^{9}$ Furthermore, it is suggested that symptoms may worsen with age, 10 due to the deterioration of cardiopulmonary function resulting from decreased compliance of the bony skeleton.

The psychological impact on patients can vary from trivial to profound, leading to social anxiety and depression. ${ }^{11}$ An aversion to undressing around others at the beach or pool is one of the most commonly described symptoms, however patients may exhibit more worrying symptoms of withdrawal.

In patients with Marfan syndrome, it is important to elucidate respiratory symptoms, which could be the result of alternative sequelae of the disease. For example, Marfan syndrome is also associated with recurrent pneumothoraces, bullae, obstructive sleep apnoea, fibrosis and emphysema. ${ }^{12,13}$

\section{Indications for treatment}

The cosmetic appearance of the pectus deformity and its psychological impact on the individual are a common indication for 
intervention. Patients often present in childhood, therefore it is imperative to elucidate whether the concerns raised are those of the patient as opposed to their parent or guardian. Where there are parental concerns, but the patient is either indifferent to the appearance or exhibits body confidence, discussion and education may be sufficient, thereby avoiding an operation.

For moderate to severe cases of pectus excavatum and carinatum not amendable to conservative management or non-operative techniques, several investigations should be considered prior to offering surgery. The severity can initially be estimated on physical examination, but should eventually be correlated with either two-view chest radiograph, limited computer tomography (CT) or full chest CT.14,15 New protocols for magnetic resonance imaging (MRI) have recently been developed which enable assessment of the Haller index without the radiation exposure of a CT scan. These can be performed within 5 minutes and do not require breath holding. ${ }^{16}$ The Haller index is utilised to assess the severity of the pectus deformity on CT (Figure 2). ${ }^{17}$ Traditionally, a Haller index of $>3.25$ was considered an indication for surgery in patients with a pectus excavatum deformity. However, more recent research suggests that the application of a correction index accounts for patients with abnormal chest morphology, thereby improving the accuracy of referral for correction. 18

For pectus carinatum, a rough clinical correlate to the Haller index is measurement of the antero-posterior (AP) at the maximal chest wall prominence compared to the lateral chest wall at this point. The closer the index is to 1.0 (i.e., a round chest dimension) the more severe the deformity.

Symptoms which commonly form part of the decision to proceed with surgery include exercise intolerance, chest pain and breathlessness. Investigations which reveal cardiopulmonary impairment further support the decision-making process. These impairments include cardiac compression or displacement, mitral valve prolapse, conduction abnormalities, pulmonary compression and restrictive lung disease.

Finally, patients who have had previous unsuccessful surgical interventions for pectus repair should also be considered for reoperation. The choice of procedure is discussed below.

\section{Guidelines for elective treatment}

Thorough history and examination remain fundamental parts of the assessment of patients with pectus deformities (Figure 3 ). Considerations should include the progression and stage of growth as well as suppleness of the chest wall. Age of the patient, height (compared to parents where possible) and timing of growth spurts should be documented. In pectus excavatum suppleness can be evaluated during clinical examination by asking the patient to perform a Valsalva manoeuvre while placing their hands on their hips and noting the degree with which the deformity is corrected. The greater the correction, the greater the flexibility of the anterior chest wall. In pectus carinatum, simply pushing on the prominence and noting how easily it reduces may provide valuable information about the appropriateness for bracing.
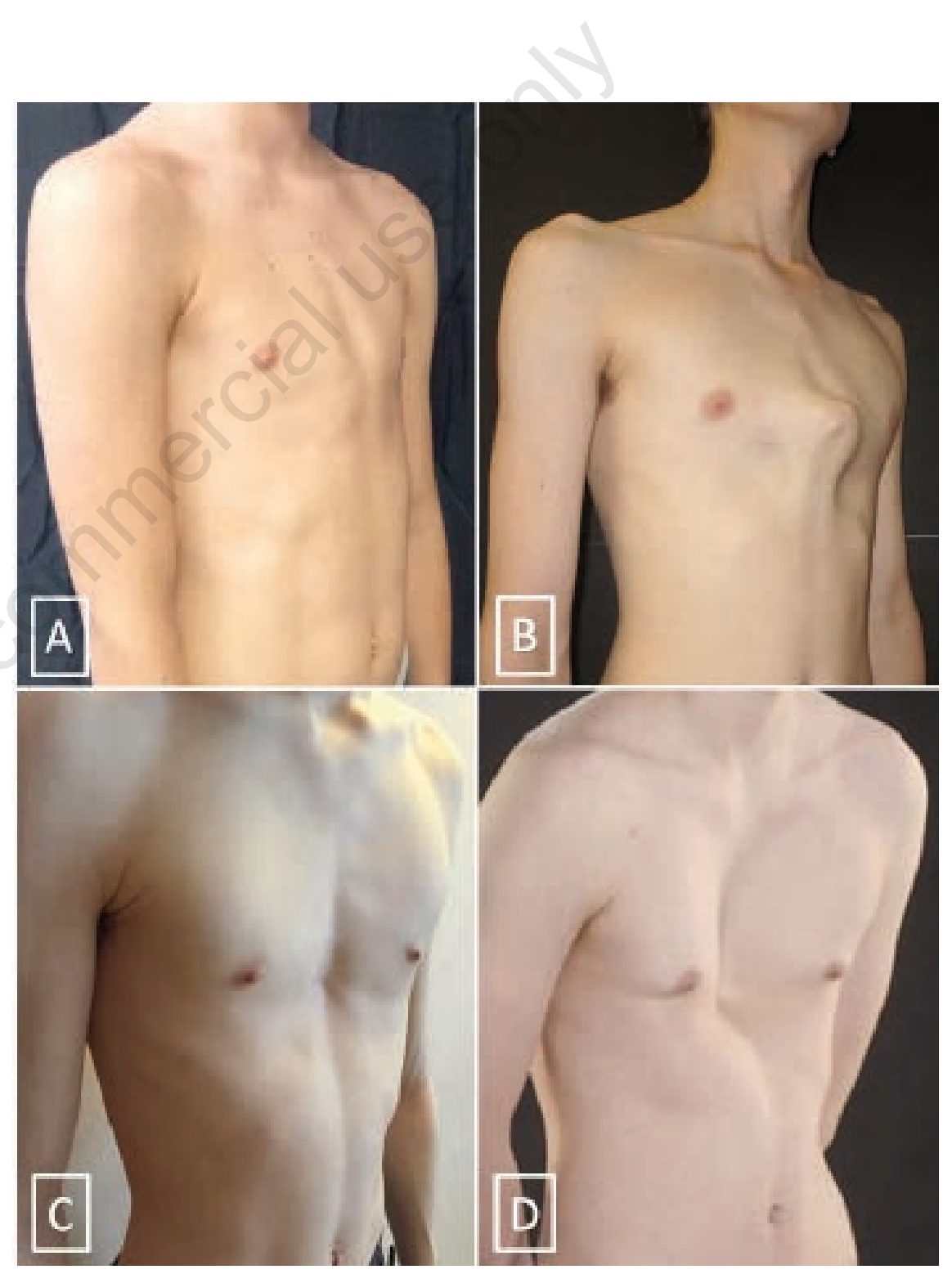

Figure 1. Mild (A) and severe (B) cases of pectus carinatum and mild (C) and severe (D)

cases of pectus excavatum.
Many patients presenting to clinic will already have a formal diagnosis of Marfan syndrome. ${ }^{2}$ In those presenting de novo, cious of the possibility of an underlying connective tissue disorder and examine carefully for other skeletal features of Marfan syndrome. If Marfan syndrome is suspected, an echocardiogram, ophthalmological examination and a genetic referral for Fibrillin-1 mutation screen should be requested as this may represent the first opportunity at diagnosis thereby enabling potentially lifesaving preventative cardiac management.

In patients with breathlessness or severe deformity, lung function tests should be undertaken to assess for a restrictive defect however, clinicians should remain suspi- 
pre-operatively, and to enable evaluation of the effect of surgical correction post-operatively. In addition to assessing aortic root dimensions with a CT scan in patients with Marfan syndrome, a transthoracic echocardiogram is essential in assessing cardiac manifestations of the disease as well as cardiac compression and impaired diastolic filling. In patients with Marfan syndrome, consideration of whether aortic root surgery is likely to be required in the future is important. Although correction of a pectus deformity can be safely undertaken both prior to or after a sternotomy for cardiac surgery, a recent paper suggests that a concomitant approach with resection of the abnormal cartilages prior to median sternotomy is associated with better intra-operative exposure and good post-operative results. ${ }^{19}$

Medical photography should be arranged to allow comparison of the preoperative and post-operative appearance, although where available, this may be supplanted by $3 \mathrm{D}$ body scanning which can help assess both the Haller index and response to treatment. 20 Pre-operative metal allergy testing should also be undertaken in any patient considered for a Nuss bar.

Finally, many patients may benefit from a formal psychological review prior to consideration of surgery. Particularly, individuals whose own assessment of the severity of their deformity does not match the clinical assessment, or those seeking a redo procedure where the initial result seems satisfactory to the clinician. Any patients exhibiting evidence of body dysmorphia or with a history of mental illness should prompt involvement of the psychiatry team before embarking on a procedure, which may have a deleterious effect on their mental health.

\section{Pectus excavatum}

\section{Traditional surgical approaches}

Surgical correction of pectus excavatum is most commonly performed using either the Nuss or Ravitch procedure.

The Nuss procedure was initially performed by Dr Donald Nuss in the 1980s, but has since been widely adopted and is now the most common method of minimally invasive repair of pectus excavatum or MIRPE. ${ }^{21}$ This approach is most commonly employed in children and young adults who have not yet reached skeletal maturity. It involves bilateral incisions in the mid-axillary line and placement of metallic bar under direct visualisation with keyhole video camera assistance. Once in place, the

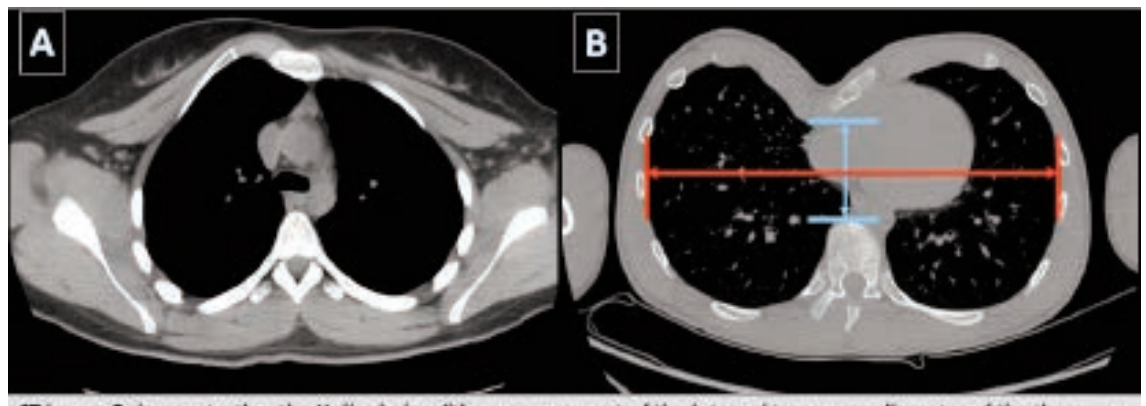

$C T$ image B demonstruting the Haller index: "The measurement of the internal tronswome diameter of the thorax on the inside of the rib cage, divided by the shartest antenopasteriar depth from the intemal aspect of the stemum of the anterior cortex of the nearest vertebral body" 2 ?

Figure 2. CT images demonstrating a patient with a pectus carinatum deformity (A) and a pectus excavatum deformity $(B)$.

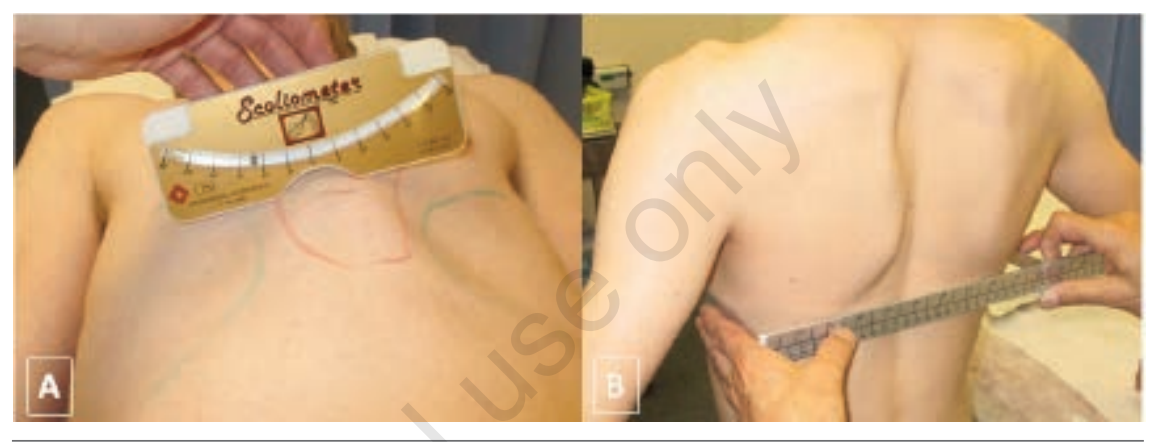

Figure 3. Examination of a pectus carinatum deformity. Measurements including the degree of sternal angulation (A) and the mediolateral chest dimensions (B).

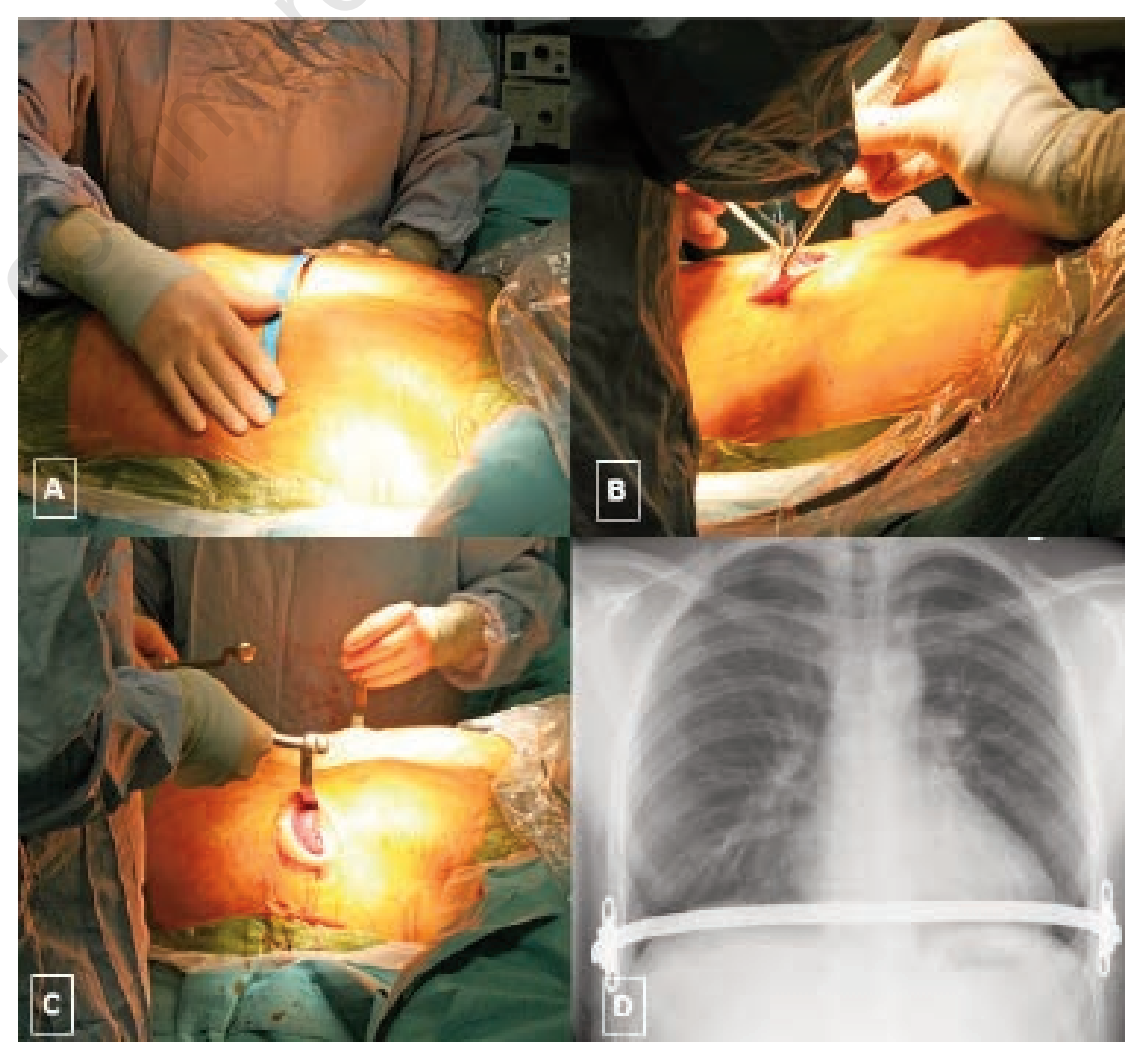

Figure 4. Minimally invasive repair of a pectus excavatum deformity with a NUSS technique. Images demonstrating: measuring of the bar (A), minimal access incision (B), the bar in situ prior to flipping (C) and a chest radiograph performed post-operatively (D). 
bar is flipped, thereby everting the deformity into a more anatomical position (Figure 4). In severe deformities or older patients, more than one bar may be required, although this is associated with an increased rate of complications. ${ }^{22}$ Routine closure of the incisions is undertaken often without the need to insert chest drains. Nuss bars typically remain in situ for at least two years and a recent review paper suggested that the older the patient, and by reflection, the stiffer the deformity, the longer the bar should remain in place. ${ }^{23}$ In patients with Marfan syndrome, surgical preference should dictate whether the Nuss bar is removed electively prior to surgery or at the time of sternotomy.

The advantages of this procedure when compared to an open Ravitch or modified Ravitch procedure include smaller incisions, less invasive surgery and reduced blood loss. However, some reports suggest that it may be associated with increased post-operative pain and longer hospital stay. 24

The Ravitch procedure was first described as costochondral osteotomy for both excavatum and carinatum. ${ }^{25}$ The underlying theory was that if all the abnormal cartilages were resected with preservation of the perichondrium, then new cartilage could grow into an anatomically corrected position. This involves either a vertical midline incision or a transverse submammary incision. A vertical incision for access may be preferred, if the deformity extends above the $4^{\text {th }}$ costal cartilage. However, patient preference should also be considered. Modifications of the procedure following complete resection of all abnormal cartilage include a sternal osteotomy to correct sternal depression and insertion of a mesh, implant or bar (Figure 5). Whereas a mesh or implant can be left indefinitely, a bar would require a further procedure for removal.

The only statistically significant advantage of a Ravitch over a Nuss procedure is a lower complication rate in older patients with stiffer deformities. ${ }^{26}$ Technically, a Ravitch allows for correction of significant sternal rotation, although this does not necessarily correlate with improved clinical outcome. This disadvantage of the Ravitch procedure involves a more extensive and longer operation with a bigger incision compared to the Nuss procedure.

Other approaches used by thoracic and paediatric surgeons include the Leonard procedure, which is similar to the Ravitch in that it describes a transverse submammary incision, excision of the lower cartilages with preservation of the perichondrium and a wedge osteotomy of the sternum. ${ }^{27}$ The
Leonard procedure differs, however, in its use of a wire which is placed behind the sternum, exiting through the skin and attached to a brace. The brace is fitted for the patient prior to the procedure and worn continuously for around three months postoperatively.

The Robicsek procedure is similar to a modified Ravitch approach with resection of the affected costal cartilage and a wedge osteotomy. 28 The sternum is corrected and fixed with a mesh which avoids the potential metalwork complications or need for bar removal experienced with alternative approaches. ${ }^{29}$

\section{Non-operative techniques}

Non-operative alternatives for the management of pectus excavatum are limited but include the use of a vacuum bell which is worn over the pectus excavatum deformity and provides suction to lift the deformity forwards. ${ }^{30}$ It is worn for several hours a day for a period of 1-2 years. Several groups have reported some good immediate results though long-term results remain unclear. 31

\section{What's new?}

Recent systematic reviews of the current data suggest that a Nuss approach is appropriate in most paediatric cases and that either a Ravitch or Nuss is safe and efficacious adult patients. ${ }^{24}$ Patient preference should be considered however, as recent studies have also suggested that many patients have strong preferences regarding whether they would prefer a minimally invasive procedure or whether they would prefer not to have metalwork in their body. ${ }^{32}$

In patients without significant sternal angulation, but past skeletal maturity, excision of the cartilage through a VATS approach has been suggested as a minimally invasive alternative to a Ravitch procedure. Additionally, procedures using minimal incisions which articulate the sternum without extensive cartilage excision have demonstrated favourable results and may reduce the operative time associated with a traditional Ravitch approach. 33

Several new approaches have been recently described. The magnetic MiniMover technique is a novel innovation developed at UCSF in San Francisco. It involves a less invasive operation whereby a magnet is inserted into the sternum and a second magnet is worn as a brace placed so that the magnetic attraction generated creates a controlled sustained pull on the breastbone. It is only offered in a very few centres but early reports suggested a good result though few centres have adopted this technique. ${ }^{34}$ An alternative, less invasive technique involving the insertion of a screw into the sternum which attaches to either an external brace, or internally thereby providing traction of the deformity is also currently in use with evidence of safety and efficacy. ${ }^{35}$

Some impressive results in the cosmesis of pectus repair have been achieved with the involvement of plastic surgeons (Figure 6). Procedures which involve implants have been demonstrated to be safe with excellent cosmetic results while also improving patients' social and emotional health. ${ }^{36}$ These approaches, however, would not be indicated alone in the presence of cardiopulmonary symptoms relating to the deformity, however, they could be used in hybrid procedures. Recent research suggests that particularly in complex pectus deformities, involvement of plastic sur-

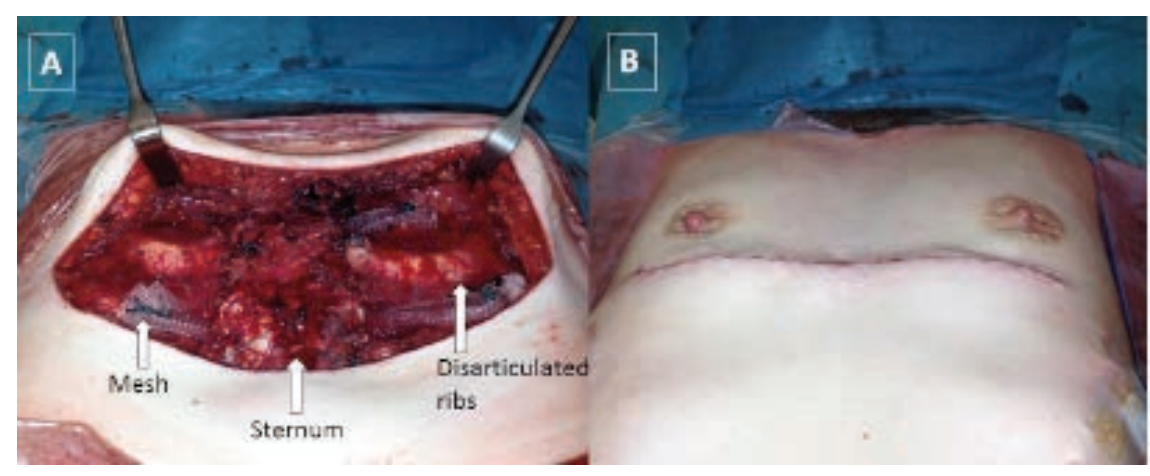

Figure 5. Open repair of a severe pectus excavatum deformity with a modified Ravitch technique. Following insertion of a mesh (A) and after skin closure (B). 
geons may improve the final cosmetic result.

\section{Pectus carinatum}

\section{Traditional surgical approaches}

A Modified Ravitch procedure for correction of a pectus carinatum deformity can be undertaken in a similar method as described above and at present is a common operative technique for surgical correction (Figure 7). The advantages and disadvantages are comparable to those with pectus excavatum deformities, with good longterm results.

A minimally invasive procedure described by Abramson et al. involves insertion of a subcutaneous bar anterior to the sternum using wires, plates and screws to configure the chest wall to an anatomical position. ${ }^{37}$ It is performed in only a few centres, with good reported results albeit in a selected group of patients with pliable pectus deformities.

\section{Non-operative techniques}

Application of a custom-made external brace is commonly utilised in the correction of a carinatum defect. This should be applied before skeletal maturity is reached and is commonly worn in a reducing regimen for at least a year. Patient concordance with treatment and success rates have been variable, however, there is a limited side effect profile and the procedure avoids all the inherent risks of surgical intervention. ${ }^{38}$

\section{What's new?}

Increasingly in flexible pectus carinatum, bracing is considered as a first-line treatment. This is supported by the Pectus Carinatum Guidelines recently published by the American Pediatric Surgical Association. Many reports now support the use of bracing methods in selected patients with pliable pectus carinatum deformity. 39

In this senior author's experience, while several bracing protocol exist, the most effective

method involves manipulation of the deformity, prior to application of a brace which maintains the reduction (Hunt 2017 unpublished data; Figure 8). The deformity is immediately reduced and thus, good compliance with bracing over the course of treatment is achieved (Hunt et al., SCTS abstract).

Bracing may also be effective as part of a hybrid procedure in older patients with a mature skeleton. These patients can be treated with a minimally invasive procedure to excise around $70 \%$ of the abnormal carti- lage in a flat ridge using with a small oscillating saw. This creates a more pliable costochondral junction, which is then amenable to bracing. This reduces the extent of surgical intervention and associated risks, however, it may cause a higher rate of dermal complications, as the brace would be applied to a surgical wound.

\section{Redo surgery}

Redo surgery can be complex and the reasons for undertaking this should be clear- ly documented prior to surgery. In complicated cases, a second opinion may be sought to ensure that the correct approach is undertaken, that correction is in the best interest of the patient and that the patient has reasonable expectations of the final cosmetic appearance.

An open operation is often considered after a previous failed procedure; however, the current literature has demonstrated that a Nuss procedure can also be successful in treating recurrence following both minimally invasive and open repair. 40 When overcorrection of a pectus excavatum results in

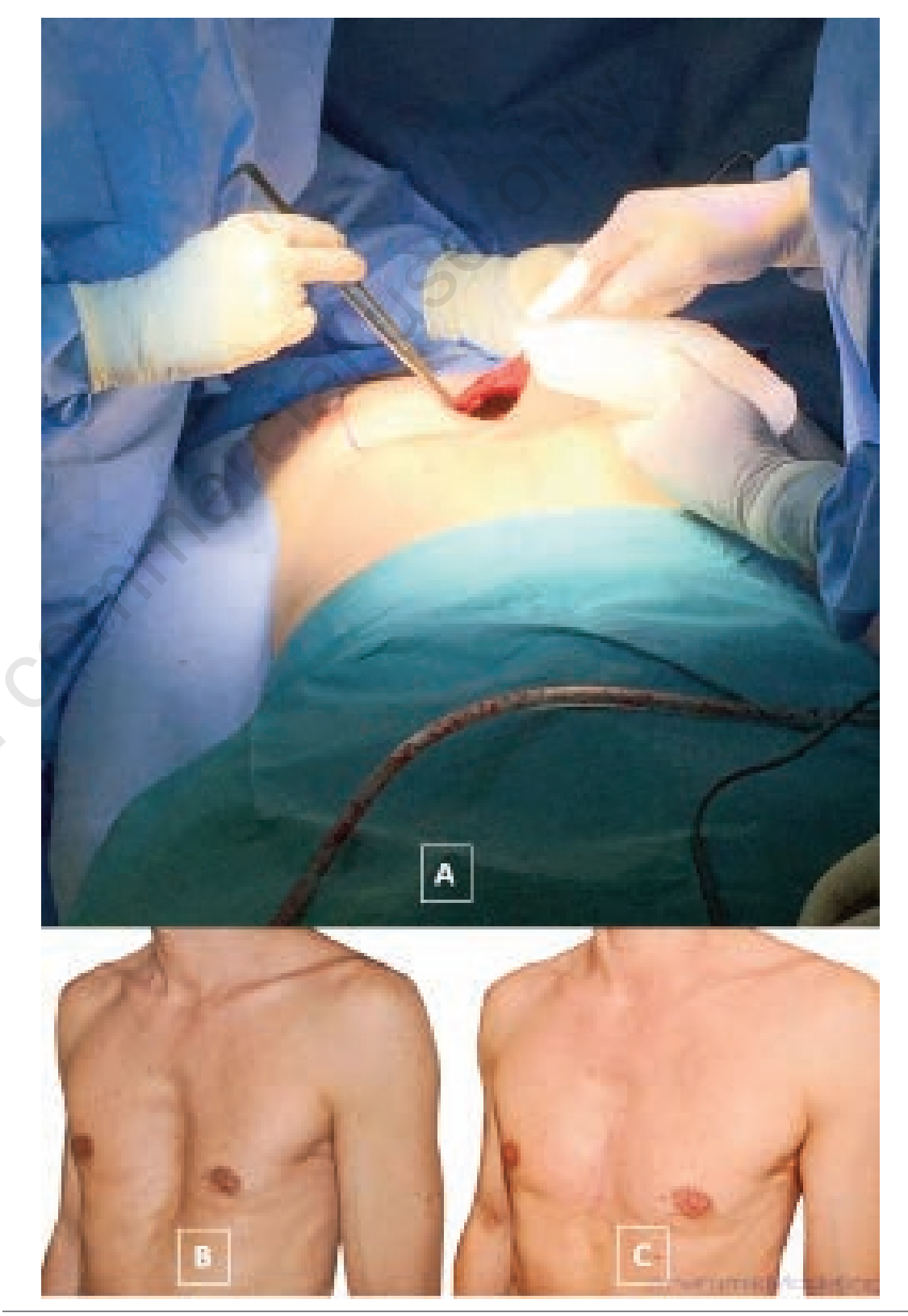

Figure 6. Insertion of a custom-made silicone implant for repair of a pectus excavatum deformity (A). Images of a pectus excavatum deformity prior to the procedure (B) and the cosmetic result following insertion of an implant $(C) .36$ 
a pectus carinatum deformity, ${ }^{41}$ a hybrid procedure can be undertaken with a minimal access modified Ravitch procedure followed by bracing.

\section{Considerations in Marfan syn- drome}

In patients with Marfan syndrome, the nature of their connective tissue disease increases the suppleness of the chest wall, and patients should be advised that they are at greater risk of recurrence following any surgical intervention. Due to this increased pliability, a longer duration of both Nuss bar and bracing should therefore be considered and some research suggests that repair should be delayed until skeletal maturity is nearly reached. 42 Open procedures can be performed as describe above, however, careful consideration of the need for cardiac surgery is required and concomitant procedures undertaken where appropriate. In patients with any underlying concern regarding aortic root dimensions, the vacuum bell technique is not advised as a safe treatment option.

In the senior author's experience, when concomitant aortic surgery and pectus correction is considered, a combined approach using a modified 'open' Nuss approach, is the treatment of choice (Figure 9). Following the completion of aortic surgery via a median sternotomy, two small lateral incisions are placed and stays placed across the chest. The sternum is then closed in the usual fashion and lifted prior to cutting the sternal wires. A Nuss is bar is then fashioned and placed under the sternum and secured. This avoids the extensive dissection of a Ravitch type procedure and reduces the risks of bleeding, instability and infection. Several bars may need to be placed, and preparations made for removal of the bars immediately following surgery should be made in case of bleeding or other immediate complications.

\section{Outcomes}

Patients should be thoroughly counselled regarding all the risks of a surgical procedure. These include: death, a cardiac event (including arrhythmia or cardiovascular collapse relating to a hypersensitivity reaction), pneumothoraces or pleural collections, pericarditis, metalwork or mesh complications (for example movement of a bar or tearing of a mesh), recurrence of the deformity, a need for a further procedure
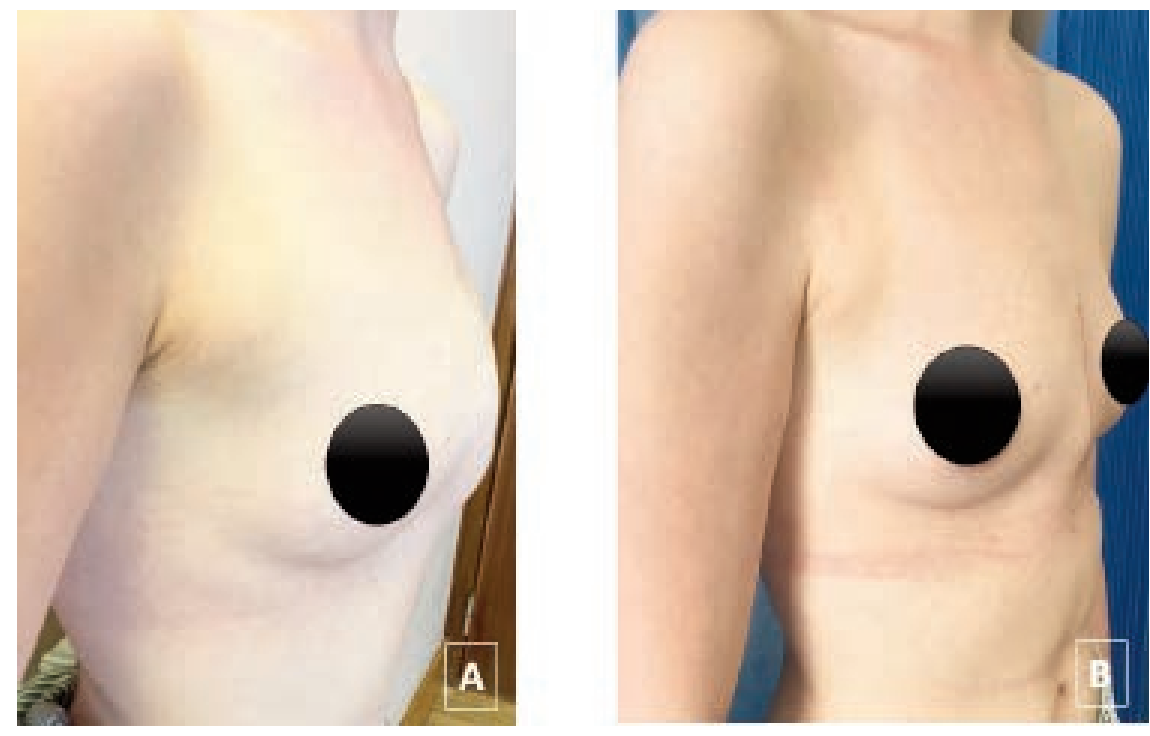

Figure 7. Pectus carinatum deformity (A) and the same patient following repair with a modified Ravitch technique (B).

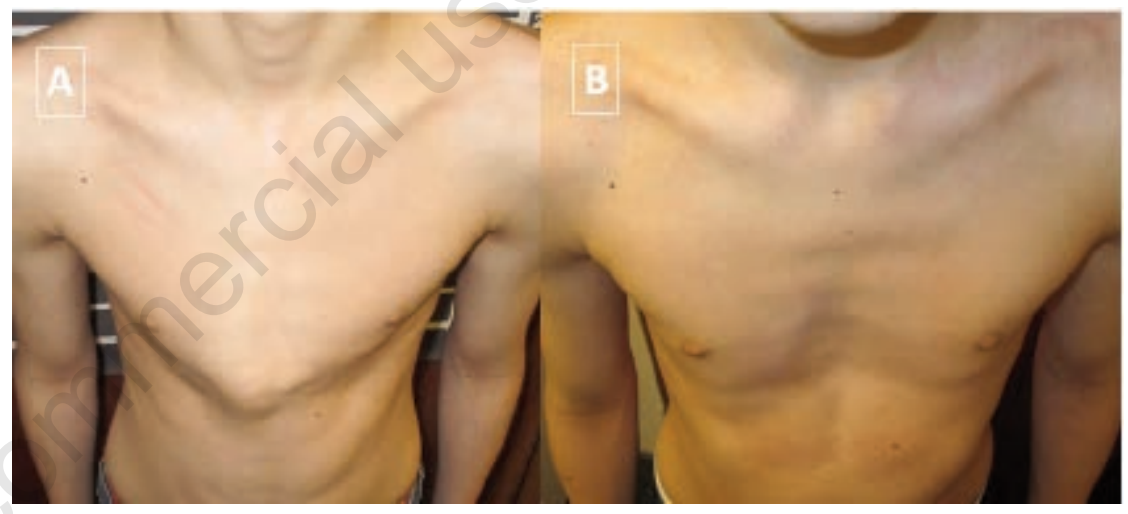

Figure 8. A central pectus carinatum deformity prior to intervention (A) and four months after manipulation and continuous external bracing (B).
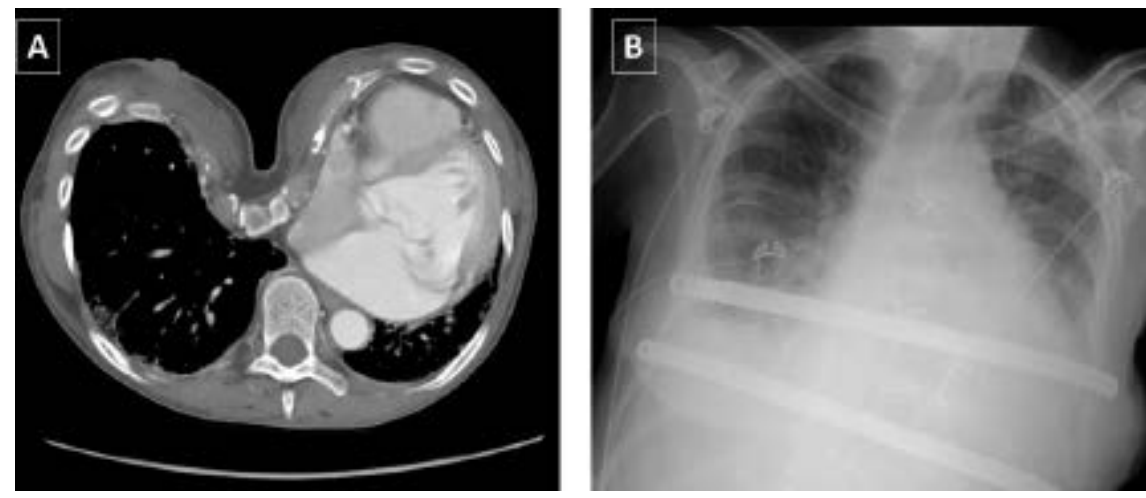

Figure 9. Pre-operative CT image of a severe pectus excavatum deformity in a Marfan patient requiring an aortic root replacement $(A)$ and following aortic surgery and placement of 2 bars concomitantly (B). 
(for example bar removal), bleeding, infection and wound problems.

Research has demonstrated short and long-term benefits with low overall morbidity following repair of a pectus deformity. Repair is associated with improved physical and psychological health of the patient and improved quality of life. ${ }^{43,44}$ It is important, however, to remember that poor self-esteem and mental health disease remain issues in this cohort to patients compared to the general population. ${ }^{45}$ There was no significant difference in patient satisfaction between the Ravitch and Nuss procedures for patients with pectus excavatum. 46

Some controversy exists regarding the impact of pectus correction on cardiopulmonary function. For example, one metaanalysis found a positive correlation between surgery for pectus excavatum and cardiopulmonary function, ${ }^{47}$ however this contradicts earlier studies which failed to demonstrate a change in physiological assessments post-operatively. 48 Many researchers, however, agree that although objective measurements, such as change in the restrictive defect have not been conclusively proven, subjectively, patients often reported an improvement in their symptoms of breathlessness and in their exercise tolerance. 49

We discuss the risks of surgery above, however, the most common post-operative issues for patients undergoing pectus repair include infection, wound problems and recurrence of the deformity. 41

\section{Recovery advice}

Patients are encouraged to mobilise early in their recovery, either later the same day or on day one post-operatively. They should expect to remain in hospital for 3-7 days following their procedure. Analgesic requirements are commonly met with patient-controlled analgesia (PCA); however, some research suggests that epidural anaesthesia provides improved pain control. 50 However, this is not recommended in Marfan syndrome as the fragility of the dura may lead to headache and the need for blood patching due to delayed dural healing.

Following discharge, patients should expect to take oral analgesia for several weeks until their pain improves. Patients are also given standard advice on discharge to seek medical attention if they become unwell. Where foreign material has been implanted, particularly a metal bar, patients should be advised to apply for a medical alert bracelet to inform healthcare profes- sionals of the need for increased force of compressions if cardio-pulmonary resuscitation were to become necessary. Similarly, patients with metal bars should be advised that an MRI would not be appropriate while the bar is in situ. Regarding a return to normal activities, common advice includes: no running or swimming for six weeks, no heavy lifting for two months and no contact sports or backpacks for three months. Finally, patients should be advised to avoid sleeping on their front for the first four weeks after surgery.

\section{Conclusions}

Anterior chest wall deformities are common in patients with Marfan and may represent the first presentation to clinicians, offering an opportunity for diagnosis, preventative management and genetic counselling. Generally, management should be tailored to the individual based on their perception of symptoms and appearance. Clinicians should also be aware of the negative prognostic impact in terms of mental health and life expectancy. Patients with Marfan syndrome and pectus deformities can present a number of challenges relating to other manifestations of the disease complicating surgical intervention, the possible need for concomitant cardiac surgery and the increased suppleness of the chest wall. However, a well-timed and successful procedure for pectus deformity can greatly improve morbidity and both operative and non-operative approaches have been demonstrated to be safe and well tolerated in this patient population.

\section{References}

1. De Maio F, Fichera A, De Luna V, et al. Orthopaedic aspects of marfan syndrome: the experience of a referral center for diagnosis of rare diseases. Adv Orthop 2016;2016:8275391.

2. Loeys BL, Dietz HC, Braverman AC, et al. The revised Ghent nosology for the Marfan syndrome. J Med Genet 2010; 47:476-85.

3. Creswick HA, Stacey MW, Kelly RE Jr, et al. Family study of the inheritance of pectus excavatum. J Pediatr Surg 2006;41: 1699-703.

4. David VL, Izvernariu DA, Popoiu CM, et al. Morphologic, morphometrical and histochemical proprieties of the costal cartilage in children with pectus excavatum. Rom J Morphol Embryol 2011; 52:625-9.
5. Torre M, Rapuzzi G, Jasonni V, Varela P. Chest wall deformities: an overview on classification and surgical options. In: Cardoso P, Ed. Topics in thoracic surgery. InTech. 2012. Available from: https://www.intechopen.com/books/top ics-in-thoracic-surgery/chest-walldeformities-an-overview-on-classification-and-surgical-options

6. Kelly RE Jr, Lawson ML, Paidas CN, Hruban RH. Pectus excavatum in a 112year autopsy series: anatomic findings and the effect on survival. J Pediatr Surg 2005;40:1275-8.

7. Wachtel FW, Ravith MM, Grishman A. The relation of pectus excavatum to heart disease. Am Heart J 1956;52:12137.

8. Park JM, Varma SK. Pectus excavatum in children: diagnostic significance for mitral valve prolapse. Indian J Pediatr 1990;57:219-22.

9. Malek MH, Fonkalsrud EW, Cooper CB. Ventilatory and cardiovascular responses to exercise in patients with pectus excavatum. Chest 2003;124:87082.

10. Kragten HA, Siebenga J, Höppener PF, et al. Symptomatic pectus excavatum in seniors (SPES): a cardiovascular problem?: A prospective cardiological study of 42 senior patients with a symptomatic pectus excavatum. Neth Heart J 2011;19:73-8.

11. Eisiedel E, Clausner A. Funnel chest. Psychological and psychosomatic aspects in children, youngsters and young adults. J Cardiovasc Surg 1999:40:733-6.

12. Wood J, Bellamy D, Child A, Citron KM. Pulmonary disease in patients with Marfan syndrome. Thorax 1984;39:780-84.

13. Neuville M, Jondeau G, Crestani B, Taillé C. [Respiratory manifestations of Marfan syndrome]. Rev Mal Respir 2015;32:173-81. [Article in French]

14. Khanna G, Jaju A, Don S, et al. Comparison of Haller index values calculated with chest radiographs versus CT for pectus excavatum evaluation. Pediatr Radiol 2010;40:1763-7.

15. Rattan AS, Laor T, Ryckman FC, Brody AS. Pectus excavatum imaging: enough but not too much. Pediatr Radiol 2010;40:168-72.

16. Marcovici PA, LoSasso BE, Kruk P, Dwek JR. MRI for the evaluation of pectus excavatum. Pediatr Radiol 2011;41:757-8.

17. Haller JA Jr, Kramer SS, Lietman SA. Use of CT scans in selection of patients for pectus excavatum surgery: a preliminary report. J Pediatr Surg 
1987;22:904-6.

18. Poston PM, Patel SS, Rajput M, et al. The correction index: setting the standard for recommending operative repair of pectus excavatum. Ann Thorac Surg 2014;97:1176-9.

19. Hysi I, Vincentelli A, Juthier F, et al. Cardiac surgery and repair of pectus deformities: When and how? Int J Cardiol 2015;194:83-6.

20. Wong KE, Gorton GE 3rd, Tashjian DB et al. Evaluation of the treatment of pectus carinatum with compressive orthotic bracing using three dimensional body scans. J Pediatr Surg 2014;49:924-7.

21. Nuss D, Kelly RE Jr., Croitoru DP, Katz ME. A 10-year review of a minimally invasive technique for the correction of pectus excavatum. J Pediatr Surg 1998;33:545-52.

22. Bilgi Z, Ermerak NO, Çetinkaya Ç, et al. Risk of serious perioperative complications with removal of double bars following the Nuss procedure. Interact Cardiovasc Thorac Surg 2016: pii: ivw322.

23. Park HJ, Kim KS. Pectus bar removal: surgical technique and strategy to avoid complications. J Vis Surg 2016;2:60.

24. Johnson WR, Fedor D, Singhal S. Systematic review of surgical treatment techniques for adult and pediatric patients with pectus excavatum. J Cardiothorac Surg 2014;9:25.

25. Ravitch MM. The operative treatment of pectus excavatum. Ann Surg 1949;129:429-44.

26. Kanagaratnam A, Phan S, Tchantchaleishvili V, Phan K. Ravitch versus Nuss procedure for pectus excavatum: systematic review and metaanalysis. Ann Cardiothorac Surg 2016;5:409-421

27. Antonoff MB, Erickson AE, Hess DJ, et al. When patients choose: comparison of Nuss, Ravitch, and Leonard procedures for primary repair of pectus excavatum. J Pediatr Surg 2009;44:1113-8.

28. Robicsek F, Watts LT, Fokin AA. Surgical repair of pectus excavatum and carinatum. Semin Thorac Cardiovasc Surg 2009;21:64-75.

29. Luzzi L, Voltolini L, Zacharias J, et al. Ten year experience of bioabsorbable mesh support in pectus excavatum repair. Br J Plast Surg 2004;57:733-40.

30. Haecker FM. The vacuum bell for conservative treatment of pectus excavatum: the Basle experience. Pediatr Surg Int 2011;27:623-7.

31. Lopez M, Patoir A, Costes F, et al. Preliminary study of efficacy of cup suction in the correction of typical pectus excavatum. J Pediatr Surg 2016;51:183-7.

32. Elsayed HH, Hassaballa AS, Abdel Hady SM, et al. Choosing between the modified Ravitch and Nuss procedures for pectus excavatum: Considering the patients's perspective. Ann R Coll Surg Engl 2016;98:581-5.

33. Fonkalsrud EW. Open repair of pectus excavatum with minimal cartilage resection. Ann Surg 2004;240:231-5.

34. Harrison MR, Gonzales KD, Bratton $\mathrm{BJ}$, et al. Magnetic mini-mover procedure for pectus excavatum III: safety and efficacy in a Food and Drug Administration-sponsored clinical trial. J Pediatr Surg 2012;47:154-9.

35. Bardají C, Cassou L. Taulinoplasty: the traction technique-a new extrathoracic repair for pectus excavatum. Ann Cardiothorac Surg 2016;5:519-22.

36. Chavoin JP, Grolleau JL, Moreno B, et al. Correction of pectus excavatum by custom-made silicone implants: contribution of computer-aided design reconstruction. A 20-year experience and 401 cases. Plast Reconstr Surg 2016;137:860e-71e.

37. Abramson H, D'Agostino J, Wuscovi S. A 5-year experience with a minimally invasive technique for pectus carinatum repair. J Pediatr Surg 2009;44:118-23; discussion 123-4.

38. Emil S, Sévigny M, Montpetit K, et al. Success and duration of dynamic bracing for pectus carinatum: A four-year prospective study. J Pediatr Surg 2017;52:124-9.

39. Lansdale: HAYES, Inc. Orthotic compression bracing for treatment of pectus carinatum. Healthcare Technology Brief Publication; 2012. Accessed from: http://www.crd.york.ac.uk/crdweb/Sho wRecord.asp? ID $=32013000263$ on 10/02/16
40. Sacco Casamassima MG, Papandria D, Goldstein SD, et al Contemporary management of recurrent pectus excavatum. J Pediatr Surg 2015;50:1726-33.

41. Swanson JW, Colombani PM. Reactive pectus carinatum in patients treated for pectus excavatum. J Pediatr Surg 2008;43:1468-73.

42. Arn PH, Scherer LR, Haller JA Jr, Pyeritz RE. Outcome of pectus excavatum in patients with Marfan syndrome and in the general population. J Pediatr 1989;115:954-8.

43. Kuru P, Bostanci K, Ermerak NO, et al. Quality of life improves after minimally invasive repair of pectus excavatum. Asian Cardiovasc Thorac Ann 2015;23:302-7.

44. Knudsen MV, Grosen K, Pilegaard HK, Laustsen S. Surgical correction of pectus carinatum improves perceived body image, mental health and self-esteem. J Pediatr Surg. 2015;50:1472-6.

45. Tikka T, Steyn R, Bishay E, et al. Short and long-term outcomes of pectus surgery. Eur Respir J 2015;46:59.

46. Lam MW, Klassen AF, Montgomery CJ, et al. Quality-of-life outcomes after surgical correction of pectus excavatum: a comparison of the Ravitch and Nuss procedures. J Pediatr Surg 2008;43:819-25.

47. Malek MH, Berger DE, Housh TJ, et al. Cardiovascular function following surgical repair of pectus excavatum: a metaanalysis. Chest 2006;130:506-16.

48. Quigley PM, Haller JA Jr, Jelus KL, et al. Cardiorespiratory function before and after corrective surgery in pectus excavatum. J Pediatr 1996;128:638-43.

49. Lacquet LK, Morshuis WJ, Folgering HT. Long-term results after correction of anterior chest wall deformities. J Cardiovasc Surg (Torino) 1998;39:6838.

50. Stroud AM, Tulanont DD, Coates TE, et al. Epidural analgesia versus intravenous patient-controlled analgesia following minimally invasive pectus excavatum repair: a systematic review and meta-analysis. J Pediatr Surg 2014;49: 798-806. 